\title{
Wissenskommunikation und Transfer für die Landschaftsentwicklung. Eine Analyse im Forschungsnetzwerk „Nachhaltiges Landmanagement ${ }^{\text {" }}$
}

\author{
Lisette Härtel • Marcel Hoffmann • Thomas Köhler • Thomas Weith
}

Online publiziert: 5. November 2015

(C) Die Autor(en) 2015. Dieser Artikel ist auf Springerlink.com mit Open Access verfügbar

Zusammenfassung Kommunikation ist eine grundlegende Voraussetzung für Transferprozesse - jedoch nicht hinreichend, um Wissen nachhaltig in der Gesellschaft zu implementieren (Johnson und Chang, Journal of Business Communication, 37(3), 238-263, 2000). Erst wenn Prozesse des Lernens hinzukommen kann relevantes Wissen nachhaltig etabliert und in Handlungen überführt werden (Larsen-Freeman, Language learning. Supplement, 63, 2013). Ziel ist, die Wirkungen der Initiierung von Forschungsnetzwerken als neuartiger Form organisationaler Intervention zu untersuchen, dabei Transfer- und Implementierungsstrategien unter der Perspektive der Wissenskommunikation, respektive des Wissensmanagements, herauszuarbeiten.

Als Forschungsfrage wird untersucht, wie Akteure der Netzwerkprojekte Prozesse des Transfers und der Implementierung von Wissen im Projektverbund gestalten. Für die empirische Befundung werden systematisch gewonnene qualitative Daten

\footnotetext{
L. Härtel, M.A. $(\bowtie) \cdot$ Prof. Dr. T. Köhler

Technische Universität Dresden,

Dresden, Deutschland

E-Mail: lisette.haertel@tu-dresden.de

M. Hoffmann, M.A.

Forum Verlag Herkert GmbH,

Merching, Deutschland

Prof. Dr.-Ing. T. Weith

Leibniz-Zentrum für Agrarlandschaftsforschung Müncheberg (ZALF) e.V.,

Müncheberg, Deutschland

Prof. Dr. T. Köhler

Fakultät Erziehungswissenschaften, Institut für Berufspädagogik und Berufliche Didaktiken,

Weberplatz 5,

Dresden, Deutschland

Prof. Dr. T. Köhler

Medienzentrum, Strehlener Straße 22/24,

Dresden, Deutschland
} 
aus Interviews mit Koordinatoren von Verbundprojekten einer Branche und zugeordneten Praxisakteuren ausgewertet.

Die Befunde betonen 1) die Gleichberechtigung von Wissenskommunikation und Organisation gemeinsamer Lernerfahrungen, weisen zudem ein 2) projektübergreifend ähnliches Begriffsverständnis von Transfer und 3) als geeignet erachtete mediale Szenarien dafür nach. Am Rande erfahren zudem 4) Prozesse der Kommunikation und des Lernens Beachtung - die als Operationalisierung von Transfer und Implementierung Anwendung in den untersuchten Netzwerken finden.

Schlüsselwörter Wissensmanagement · Landnutzungswandel · Kommunikation · Netzwerkorganisation

\section{Knowledge communication and transfer for the landscape development. An analysis of the research network "Sustainable Land Management"}

Abstract Communication is a fundamental prerequisite for transfer of processes, but not sufficient to sustainably implement knowledge in the society (Johnson und Chang, Journal of Business Communication, 37(3), 238-263, 2000). Only if processes of learning are added, relevant knowledge can be converted into actions and will become effective (Larsen-Freeman, Language learning. Supplement, 63, 2013). The aim is to investigate the effects of the initiation of research networks as a novel form of organizational intervention, thereby transfer and implementation strategies from the perspective of Knowledge Communication, respectively of knowledge management to work out.

As a research question is examined, how actors of network projects design processes of transfer and implementation of knowledge in their project network. For the empirical investigation qualitative data is systematically obtained from interviews with coordinators of joint projects and associated practice players of a single domain and evaluated. The findings emphasize (1) the equality of knowledge communication and organization of joint learning experiences, moreover, have an (2) across projects similar conceptual understanding of transfer and (3) consider similar media scenarios as appropriate. Marginally also (4) processes of communication and learning receive attention-which are used as operationalization of transfer and implementation in the studied networks.

Keywords Knowledge management - Land use change ' Communication · Network organization

\section{Einleitung: Wissenserzeugung und -akteure im Forschungsverbund}

Die Ausweitung der Ressource Wissen ist das prägende Merkmal der modernen postindustriellen Gesellschaft. Wissen ist die zentrale Ressource, um als Individuum, Gruppe oder Organisation zu agieren (Hasler Roumois 2010). In einer Wissensgesellschaft hängen immer mehr Prozesse vom Umgang mit Wissen ab. Der rasche Wandel 
und die dadurch bedingte geringe Halbwertszeit von Wissen machen strategische Selektionsprozesse ebenso notwendig wie Strategien für das Bewahren und Nutzen dieses Wissens. Die Wissensgesellschaft verändert sich mit sich innovierenden sozio-technologischen Formen weiter. Dazu gehören insbesondere virtuelle Organisationen bzw. hybride Mischformen zwischen klassischen Präsenzgemeinschaften und deren online-gestützten Pendants (Baum et al. 2003). Die vom deutschen Bundesministerium für Bildung und Forschung (BMBF 2013) finanziell unterstützte Fördermaßnahme „Nachhaltiges Landmanagement (NLM)“ mit seinen aktuell 25 Projektverbünden ist hier ein typisches Beispiel (Kaiser et al. 2012). Mit dem Ziel der zukunftsweisenden Gestaltung der Forschungslandschaft am Übergang zwischen Anwendungsforschung und Systemgestaltung werden durch das BMBF bewusst netzwerkartige Strukturen in Form von Projektverbünden aus Forschung und Praxis adressiert. Neuartig ist dieser Ansatz insofern, als dass neben dem Interesse am fachbezogenen Erkenntnisgewinn im Themenfeld der Landnutzungsforschung auch die Frage des Wissenstransfers analysiert wird. Dies geschieht insbesondere durch die domänenspezifische Kompetenz der hier im Fokus stehenden Raum-, Umwelt- und Planungswissenschaften sowie der Landschaftsforschung (vgl. Zscheischler et al. 2014; Küster 2012; Salet 2014), bezieht zudem Expertise aus angrenzenden Bereichen wie z. B. der Klimaforschung (vgl. Knieling und Müller 2015; Knieling und Roßnagel 2015) oder der Forstwissenschaft (vgl. Janse 2007) ein.

Der strukturelle und inhaltliche Aufbau der Fördermaßnahme „Nachhaltiges Landmanagement" wird in den nachfolgenden Abb. 1 und 2 veranschaulicht. Die

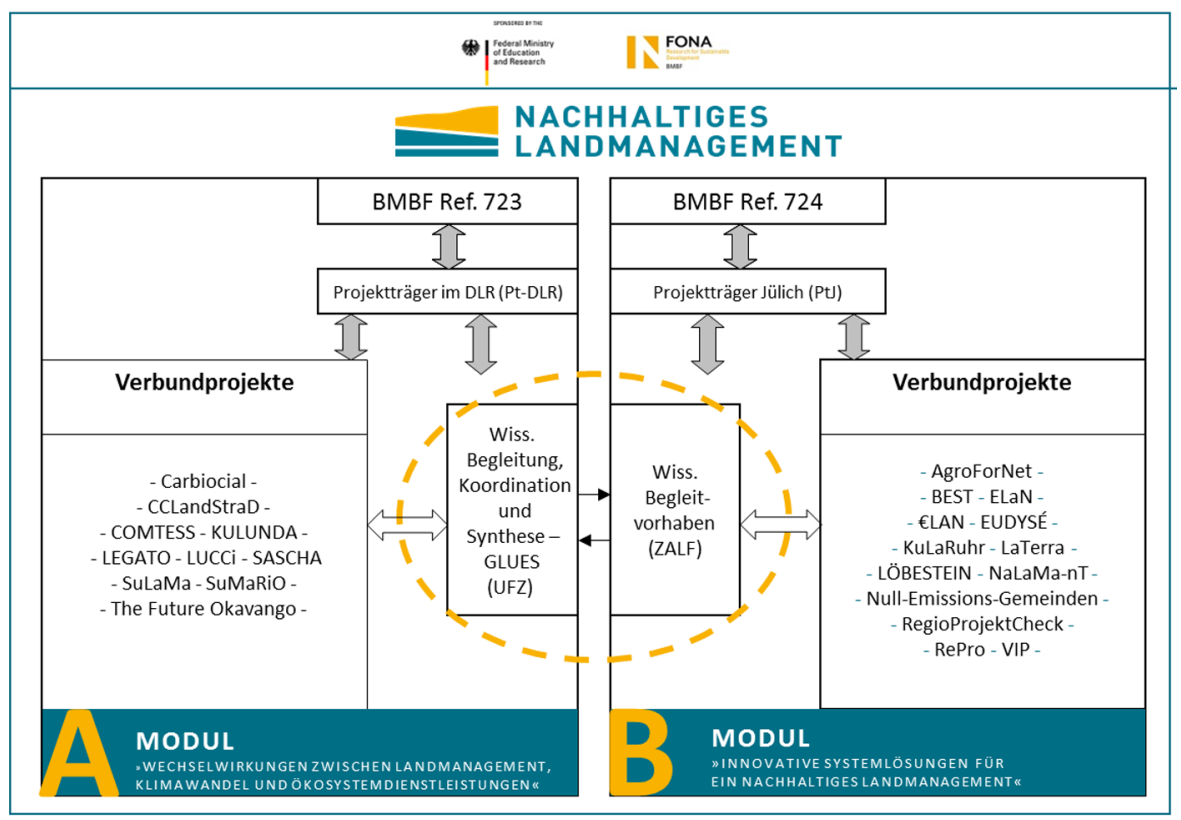

Abb. 1 Struktur des Förderprogramms „Nachhaltiges Landmanagement“ (eigene Darstellung, wobei die Akronyme jeweils für einzelne Verbundprojekte stehen) 


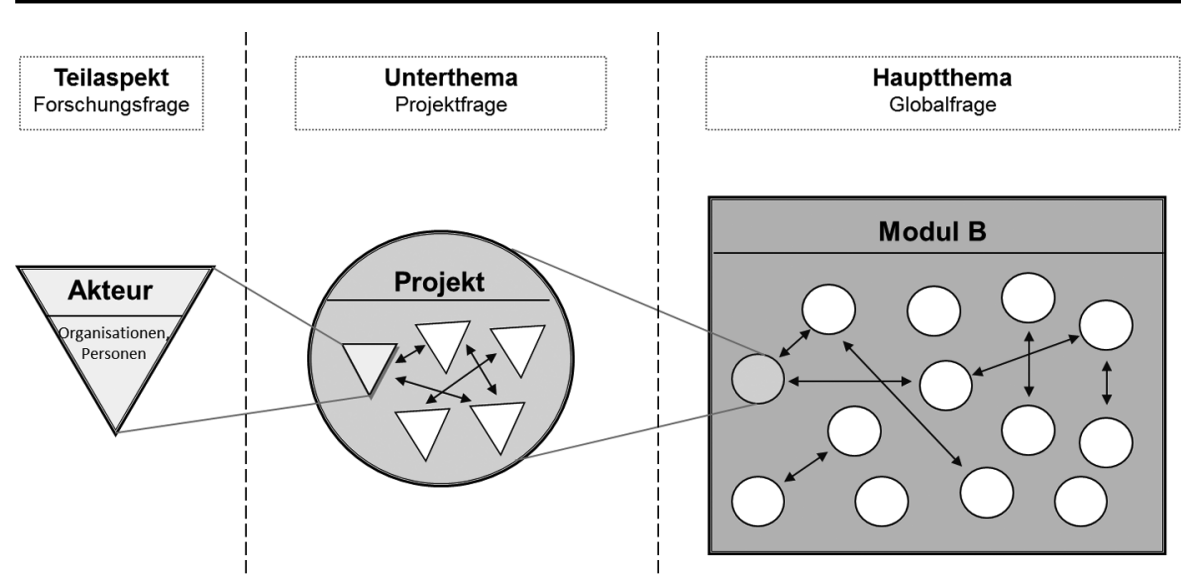

Abb. 2 Schematische Darstellung der Netzstruktur von Modul B und seiner Projekte (vgl. Härtel und Hoffmann 2013)

Maßnahme gliedert sich in das Modul „Globale Wechselwirkungen“ (A). ${ }^{1}$ sowie das Modul „Innovative Systemlösungen“ (B), welches mit seinen 13 deutschland und europaweit vernetzten Projekten das Forschungsobjekt des vorliegenden Aufsatzes ist. Unter Einbezug regionaler Akteure und Adressaten verfolgen die dem Modul B zugeordneten Projekte eine transdisziplinäre Herangehensweise.

Die Bearbeitung verschiedener Fachthemen und Forschungsfragen erfolgt in untergeordneten Arbeitspaketen der 13 Verbundprojekte sowie im wissenschaftlichen Begleitvorhaben. Infolgedessen entstand eine Netzstruktur, nicht nur innerhalb des Moduls B, sondern auch innerhalb der Verbundprojekte. Hier werden die Aufgaben unter Einbezug vielfältigster Akteure (Personen aber auch Organisationen etc.) bewältigt. Der Aufsatz betrachtet somit Transfer und Implementierung in Netzwerken, wobei die Maßnahmen der einzelnen Projekte im Fokus stehen.

Transfer und Implementation finden hierbei - entsprechend der Ausschreibungsvorgaben des BMBF - unter dem Anspruch transdisziplinären Handelns statt. Transdisziplinarität wird als Arbeits- und Lernform mit wechselseitigem (Wissens-) Austausch von Wissenschaft und Praxis verstanden. Als zentrale Komponenten werden Orientierung an gesellschaftlichen Problemstellungen, Ziel- und Handlungsorientierung, offene (nichtlineare) Forschungsprozesse, Integration von Interdisziplinarität sowie Reflexivität der Prozessgestaltung angesehen (vgl. Zscheischler et al. 2014; Zscheischler und Rogga 2015).

Beide Module werden durch je ein wissenschaftliches Begleitvorhaben ergänzt. Die Begleitvorhaben unterstützten den Austausch zwischen den Verbundprojekten, ohne eine eigenständige zusätzliche Organisationsform darzustellen oder aufzubauen. Mittels Analysen der Innovationsprozesse und deren Gestaltungsfähigkeit im Landmanagement wollen die Begleitvorhaben übertragbare und anwendbare Lösungen erarbeiten (Kaiser et al. 2012). Sowohl in ihren Beratungs- und Koordinierungsaufgaben als auch bei den eigenen Analysen und Synthesen beziehen sie sich auf die Aktivitäten der Verbundprojekte. Um die Wirkungen der neuartigen, netzwerkbasierten Zusammenarbeit zu untersuchen stellen die Autoren die Frage, wie die Projekte 
Transfer- und Implementierungsstrategien unter der Perspektive der Wissenskommunikation, respektive des Wissensmanagements, gestalten. Im Fokus stehen dabei die Prozesse der Kommunikation und des Lernens, die als operationalisierte Methoden des Transfers und der Implementierung ihren Einsatz finden. In Anlehnung an bisherige Forschungen (vgl. Zscheischler et al. 2012; Rogga et al. 2014) können die Begrifflichkeiten Transfer und Implementierung wie folgt definiert und im Sinne des Wissensmanagements interpretiert werden, wobei die Diskussion in der Fachliteratur noch nicht abgeschlossen scheint (vgl. Rogga et al. 2014):

- Transfer ist der Prozess der Übermittlung von einem Sender an einen Empfänger (Kreitel 2008). Gegenstand der Übermittlung ist das innerhalb eines Projektes erzeugte Wissen. Der beidseitig gerichtete Prozess des Wissensaustauschs gründet dabei auf Kommunikation. Transfer beschreibt die zielorientierte und zweckdienliche Übermittlung von explizitem Wissen an unterschiedliche Wissensnachfrager. Als Informationsfluss ist er dabei tragendes Moment für die Steuerung und Organisation der Wissensentstehung, damit eine nutzenrelevante kollektive Wissensbasis geschaffen werden kann.

- In der Implementierung spiegeln sich die individuellen Aktivitäten und Prozesse bei der Übernahme des neuen Wissens durch Personen aus der Zielgruppe sowie dessen danach folgende Nutzung wieder. Die dabei sattfindenden Internalisierungsprozesse sind notwendig, da eine reine Vermittlung von Informationen noch kein Wissen entstehen lässt. Implementierung ist somit die (langfristige) Umsetzung des Wissens, dargestellt durch Lernprozesse. Die Brücke zwischen Transfer und Implementierung bildet die Anwendung in der Gestalt, dass hier Wissen operationalisiert wird und bereitgestellte Informationen kontextspezifisch genutzt werden, wobei dies vorerst in einem kurzfristigen Zeitrahmen geschieht. Mit der Verstetigung wird letztlich das Ziel der Implementierung erreicht.

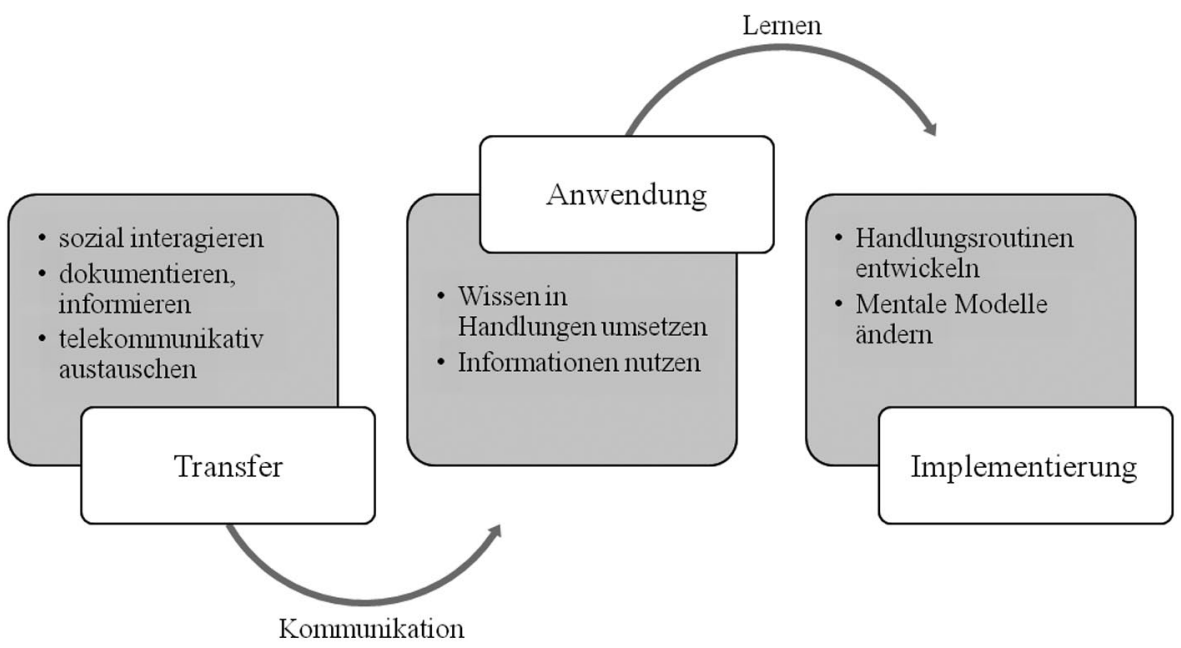

Abb. 3 Zyklus Transfer-Anwendung-Implementierung (vgl. Härtel und Hoffmann 2013) 
Die Nachfolgende Abb. 3 veranschaulicht das Zusammenspiel von Transfer, Anwendung und Implementierung:

Für die Projektakteure ergeben sich die Handlungsfelder der Kommunikation und des Lernens, welche in ihren Merkmalen im Theorieteil beschrieben werden, ergänzt durch Hinweise auf strategisches Vorgehen. Insbesondere wird auf die Netzwerkforschung eingegangen.

\section{Theoretische Grundlagen: Wissenskommunikation als Transfermethode}

\subsection{Kommunikation und Lernen}

In Anlehnung an das klassische Kommunikationsmodell kann Kommunikation als der Hauptprozess des Transfers betrachtet werden, d. h. das Medium, über welches Informationen und Wissen vom Wissensanbieter (Sender) zum Wissensnachfrager (Empfänger) transportiert werden (Herbst 2000). Im Wissensmanagement hat Kommunikation grundsätzlich drei Aufgaben (ebd.): Kommunikation 1) ermöglicht, interne und externe Zielgruppen über die Bedeutung des Wissens im Projekt zu informieren, 2) dient der Verständigung der Beteiligten und 3) transportiert das Wissen selbst. Wird dieses kommunizierte Wissen in Handlungen umgesetzt und führt es langfristig zu Veränderung bestehender kognitiver Muster der Individuen durch Integration des selbigen Wissens und der Informationen, welche bei einem Transfer ausgetauscht werden, kann vom Prozess des Lernens gesprochen werden. Insofern findet beim Lernen in Folge der Kommunikation eine Implementierung statt. Als Analyserahmen würde sich ggf. auch einen Ko-Konstruktions-Perspektive, die stärker die subjektive Bewertung des kommunikativen Handelns untersucht - vgl. Gergen (2009), Camargo-Borges und Rasera (2013) - den die Autoren aber an anderer Stelle aufgreifen (Kaiser et al. 2015).

In Projekten ist vor allen Dingen das Zusammenbringen und die Kombination einzelner Teammitglieder und Stakeholder und deren Kompetenzen eine Methode, Kommunikation und Lernen effizient zu gestalten (Probst et al. 1998). Die Akkumulation des Wissens einzelner ermöglicht eine produktive Projektarbeit, wodurch schnelle und umfängliche Problemlösungen möglich sind, die allein durch individuelle Fähigkeiten und Wissensbestände nicht gefunden werden können (ebd.). Gerade im Hinblick auf das Wissensmanagement in Projekten kann man das Lernen aus der Gruppenperspektive betrachten. Gruppen- oder Teamlernen wird als „Veränderungsprozess auf Basis gruppeninterner Kommunikation und Interaktion verstanden“ (Oberschulte 1996). Dadurch, dass Projekte meist durch eine flache Hierarchie charakterisiert sind, kann auf individueller Ebene, aber auch zwischen Funktionsbereichen schnell und zielstrebig miteinander interagiert werden. Förderlich ist dabei eine dichte Vernetzung (lokal oder virtuell) der Projektakteure, worüber ein gezielter Informations- und Wissensaustausch stattfinden kann, der durch gemeinsame Ziele des Teams begünstigt wird und die Grundlage für Lernaktivität ist (Prokesch 1997). In welcher Art und Weise und mit welcher Intensität Kommunikation und Lernen in Projekten stattfindet, ist der Untersuchungsgegenstand des vorliegenden Aufsatzes. Von besonderem Interesse sind dabei das Verständnis und das Bewusstsein von Kom- 
munikations- und Lernprozessen bei den Projektbeteiligten im Verbund des Nachhaltigen Landmanagements.

\subsection{Netzwerkforschung}

Zentrales Thema der Netzwerkforschung ist die Zusammenarbeit von Personen und Institutionen außerhalb klassischer, hierarchischer Organisationen. Diese erstreckt sich auch auf virtuelle Gemeinschaften, wo digitale Werkzeug bspw. im Wissensmanagement betrachtet werden. Grundlage dieser Kooperation ist die individuelle Handlungsbereitschaft zum Austausch von Informationen mit dem Ziel, soziale Synergieeffekte aus der Interaktion der Teilnehmer zu erzielen. Einsatzgebiete der Netzwerkforschung sind ausgesprochen vielfältig, reichen auch in der Zeit vor der Nutzung des Internet bereits von der Sozial-, Politik-, Wirtschafts- bis hin zur Computer-, Informations- und Neurowissenschaft, etc. (Freeman 2006). Aktuell werden soziale Netzwerkdaten bevorzugt mithilfe spezieller relationaler Softwaretools erhoben, visualisiert und analysiert. Insbesondere Kollaboration in digitalen Wissensund Lernnetzwerken und die damit einhergehende Kommunikation von Teilnehmern sind ein viel beachtetes Forschungsthema (Stützer et al. 2012).

Der Begriff des Wissensnetzwerks wird in der Literatur vielseitig verwendet (vgl. ebd.), wobei uneinheitliche Konzepte existieren. Hierzu zählen unter anderem der Communities of Practice-Ansatz von Lave und Wenger (1998) sowie der Begriff der Wissensgemeinschaft, unter anderem von North et al. (2000). Wissensnetzwerke können allgemein als soziale Netzwerke verstanden werden, die durch IKT unterstützt und durch soziale Interaktion mobilisiert werden. Bei Forschungsverbünden handelt es sich um soziale Netzwerke. Mitunter werden diese auch als Forschungsnetzwerke bezeichnet und direkt in den o. g. Funktionsweisen adressiert - vgl. das „e-Science Forschungsnetzwerk Sachsen“ (www.escience-sachsen.de) nebst dem zugehörigen Förderprogramm „Forschungsnetzwerke“ des Freistaates Sachsen 2011-2014.

\section{Forschungsmethodischer Zugang}

\subsection{Forschungsfrage}

Forschungsverbünde und -netzwerke sind seit einiger Zeit im Fokus der Forschungsförderung - wie auch eine sich verändernde Perspektive auf die Schaffung gesellschaftlicher Innovation (Hiltz und Turoff 1993; UFZ 2009; UFZ 2011). Forschungsverbünde unterscheiden sich vom klassischen Sonderforschungsbereich insbesondere dadurch, dass sie häufig inter- oder transdisziplinäre Perspektiven verfolgen. Hinzu kommt die ungewöhnliche organisationale Konfiguration, die weniger verbindlich als eine klassische Matrixorganisation, jedoch deutlich umfangreicher als ein einzelnes Projekt ist (BMBF 2013). Oft lässt sich ein solcher Verbund in eine Zahl kleinerer, lose gekoppelter Teilprojekte gliedern. Die nachfolgende Untersuchung betrachtet eben diese Mikroebene. Die einzelnen Projekte werden als Netzwerke und Verbünde auf kleinster organisationaler Ebene verstanden. Sie sind charakterisiert durch eine langfristige, auf Freiwilligkeit beruhende Zusammenarbeit. Die Koope- 
rationsprozesse (Transfer und Implementierung) erfolgen dabei sowohl innerhalb als auch übergreifend zu anderen, im Verbund organisierten Projekten. Die Forschungsfragen, wie sich hier die Wissensprozesse Kommunikation, als Methode des Transfers und das Lernen als operationalisierte Handlung der Implementierung gestalten, sollten im Zuge der empirischen Untersuchung in ihrer Ausprägung innerhalb von best-practice-Projekten erhoben werden. Als geeignete Methode für die Datenerhebung bieten sich Interviews mit Projektakteuren an. Diese wurden dazu angeregt, ihre bisherigen Strategien des Wissensaustauschs und der Verstetigung reflektierend zu beschreiben. Auf dieser textlichen Grundlage lassen sich schließlich Aussagen über Strategien des Transfers und der Implementierung ableiten.

Aus der bisherigen Forschungslandschaft wurden die Angaben über Transfer- und Implementierungsstrategien in einem Projektverbund aufgegriffen (vgl. z. B. Reid et al. 2009; Stringer und Dougill 2013). Als Grundlage für die Forschungsfrage werden 1) die Hauptprozesse des Transfers und der Implementierung in ihren Handlungsausprägungen der Kommunikation und des Lernens projektkohärent beschrieben und 2) thematisiert wie die Projektakteure Transfer- und Implementierungsprozesse von Wissen in der Fördermaßnahme „Nachhaltiges Landmanagement“ gestalten. In der weiteren Differenzierung geht es auch um deren Begriffsverständnis, deren Strategien und die verwendeten Tools. Transfer und Implementierung können kontextspezifisch unterschiedlich belegt sein. Im Verständnis der Autoren ist Transdiziplinarität die Abfolge von Transfer-Anwendung-Implementierung (vgl. Kap. 1). Um herauszufiltern, welche Definitionen die Akteure den Begriffen zugrunde legen und welche Maßnahmen sie in der Konsequenz ergreifen, werden sie nach ihrem Begriffsverständnis gefragt. Dadurch können auch die Strategien und Tools eingeordnet werden,

Tab. 1 Forschungsfrage mit Untersetzung und Antworterwartung

Forschungsfrage: Wie gestalten Akteure im Projektverbund „Nachhaltiges Landmanagement“ die Transfer- und Implementierungsprozesse von Wissen?

Untersetzung: Was verstehen die Projektakteure unter Transfer und Implementierung?

Antworterwartung: Projekte verstehen Transfer als kommunikativen, wechselseitigen Austausch zwischen Projektakteuren. Implementierung ist für sie die praktische Umsetzung der Ergebnisse und Lösungen aus dem Forschungsprozess, geht für die Projekte einher mit Wissensentwicklung und Lernen Untersetzung: Welche Strategien werden angewendet, um Wissen zu transferieren und zu implementieren?

Antworterwartung: Für einen kontinuierlichen wechselseitigen Wissenstransfer haben die Projekte Regeln formuliert, um nicht nur ihre Ergebnisse, sondern auch den Prozess der Forschung und Entwicklung von Erkenntnissen transparent zu gestalten und um voneinander zu lernen. Transfer im Sinne von Kommunikation und Implementierung, im Sinne von Lernen, erfolgen kontinuierlich und prozessbegleitend. Systematische Strategien liegen jedoch nicht vor, Kommunikation und Lernen finden ad hoc und situationsspezifisch statt. Ein Wissensmanagement mit konkret formulierten Anforderungen an die Projekte und deren Akteure gibt es nicht, wird aber unbewusst angewandt

Untersetzung: Mithilfe welcher Tools werden Transfer und Implementierung unterstützt?

Antworterwartung: Es wird erwartet, dass vor allem face-to-face Kommunikation beim Transfer eingesetzt wird. Digitale Tools finden häufig Einsatz, aufgrund der räumlichen Trennung der Projekte und Akteure, die damit aufgehoben werden kann. Es wird nicht erwartet, dass es explizit ausgearbeitete Lernarrangements gibt. Dennoch werden unbewusste Gelegenheiten vermutet, bei denen Lernen stattfindet (z. B. Messen, Workshops) und Implementierung erzielt wird 
da es sich um prozessorientierte Begriffe handelt. Tabelle 1 stellt die Forschungsfrage mit Unterfragen kurz dar:

\subsection{Forschungsdesign und Stichprobe}

Als empirische Umsetzung wurden aus verschiedenen best-practice-Projekten Projektakteure über ein leitfadengestütztes, problemzentriertes Interview zu Transferund Implementierungsmaßnahmen befragt. In der Auswertung des Materials konnten mittels der qualitativen strukturierten Inhaltsanalyse nach Mayring (2010) Handlungsweisen bezüglich der Kommunikation und des Lernens herausgearbeitet und mit Transfer und Implementierung in Verbindung gebracht werden. Diese empirischen Befunde zur Vorgehensweise sind Grundlage für die Diskussion und den Rückbezug der Ergebnisse auf die eingangs behandelte Theorie.

Befragt wurden 2 Projekte mit 8 Interviewpartnern innerhalb von 5 Interviews. Es ergibt sich somit eine Stichprobengröße von $N=8$. Der Auswahl des Samples wurden verschiedene Indikatoren zugrunde gelegt. So ist der erste Indikator für die Auswahl der Projekte die innovative Ausrichtung deren Handlungsfelder. Besonderes Augenmerk wird auf Transdisziplinarität und Partizipation von Praxisakteuren gelegt indem die Untersuchung ausschließlich solche Projekte analysiert, bei denen Wissenschaft und Praxis tatsächlich kommunizieren und kooperieren. Schließlich soll mit der Erhebung vorrangig gezeigt werden, wie und unter welchen Bedingungen Transfer und Implementierung auf systematischen Weg zustande kommen. Dabei orientiert sich die Befragung in erster Linie an best-practice-Lösungen. Bei der Übertragung der gelungenen Transfer- und Implementierungsstrategien auf andere Projekte ist aber zu beachten, dass die dazugehörigen Prozesse in hohem Maße situations- und kontextspezifisch sind. Daher gestaltet sich eine Übertragbarkeit im Sinne von Musterlösungen schwierig.

Für die Untersuchung der Forschungsfrage nach Transfer- und Implementierungsstrategien von Wissen im Projektverbund wurden für den vorliegenden Aufsatz 5 Interviews aus dem Verbundprojekt „Nachhaltiges Landmanagement“ ausgewählt. Koordinatoren, Schnittstellenperson zur Praxis als auch direkte Praxisvertreter wurden zu unterschiedlichen Terminen vor Ort oder online über Adobe Connect interviewt. Die hier ausgewählten Personen repräsentieren prototypisch Projekte im Forschungsnetzwerk ,Nachhaltiges Landmanagement“" und erfüllen so die Anforderungen an die Übertragbarkeit auf das gesamte Netzwerk.

\subsection{Erhebungsmethode}

Sozialwissenschaftliche Forschungsgegenstände, wie im vorliegenden Fall die Fördermaßnahme „Nachhaltiges Landmanagement“ mit seinen Akteuren, sind durch einen komplexen und prozessualen Kontext charakterisiert (Witzel 1985). Bereits die Begleitforschung zur BMBF Förderung hatte festgestellt, dass es einen Klärungsbedarf in Bezug auf die Kommunikationsstrukturen der einzelnen Projekte gibt (Kaiser et al. 2012). Um im Sinne der Forschungsfrage an beispielhafte Informationen über Transfer und Implementierungsstrategien innerhalb des Projektverbundes zu kommen, wurde als Erhebungsmethode das problemzentrierte Interview ausgewählt. In 
der vorliegenden Untersuchung geht es um die Wahrnehmung der spezifischen Problemlage der Fördermaßnahme „Nachhaltiges Landmanagement“", repräsentiert durch einzelne Akteure im Verbundprojekt. Das Erkenntnisinteresse erstreckt sich gleichermaßen auf die projektinternen wie -externen Transfer- und Implementierungsstrategien. Als Operationalisierung für eine solche Fragestellung bieten sich neben einer Fallanalyse insbesondere Interviews mit den handelnden Akteuren an, umso deren Erfahrungen entsprechend der Fragestellungen zu erheben und eine systematische Wissensbasis aufzubauen wobei die in der Netzwerkforschung etablierte Methodik der Analyse von Interaktionsbeziehungen und der Bewertung dieser in struktureller Sicht nicht im Zentrum des Beitrages stehen sollte. Vielmehr geht es um die durchaus subjektive Bewertung des Kommunikations- und wissensbezogenen Verhaltes der Akteure in ihren Verbünden und Projekten. Eine solche Netzwerkanalyse im engeren Sinne wäre wünschenswert, die Autoren haben dies auf eine prinzipielle Abstraktion (vgl. Abb. 2) begrenzt. Im Forschungsverlauf wurde als Grundlage für die Gespräche ein Leitfaden entwickelt, der sowohl inhaltliche als auch kommunikationsbezogene Anteile umfasst. Diese Gemeinschaftsproduktion aller Forscher des wissenschaftlichen Begleitvorhabens im Projektverbund „Nachhaltiges Landmanagement“ deckt alle interessierenden Themenbereiche hinsichtlich Transfer und Implementierung ab. Für die vorliegende Auswertung erfolgte eine Fokussierung auf die Transfer-, d. h. kommunikationsbezogenen Aspekte.

\subsection{Auswertungsmethode und Gütekriterien}

Für die Auswertung der Interviews wird auf die qualitative Methode der Inhaltsanalyse nach Mayring zurückgegriffen (Mayring 2010). Diese ermöglicht die Analyse kommunikativen Datenmaterials. Um die Masse an Text zu bewältigen und um der Erhebungsmethode des problemzentrierten Interviews gerecht zu werden, wurde die Strukturierte Inhaltsanalyse (vgl. ebd.) als Hauptauswertungsmethode ausgewählt. Auf der Grundlage theoriegeleitet gebildeter Kategorien wird das transkribierte Material systematisch durchgearbeitet und entsprechende Textstellen zugeordnet.

Mittels Fokussierung auf Transfer und Implementierung von Wissen im Projektverbund und die daraus resultierende Frage nach den Erscheinungsformen in der Praxis können entlang der Theorie auf deduktivem Weg Haupt- und Unterkategorien gebildet werden, die das Forschungsinteresse eingrenzen (vgl. Härtel und Hoffmann 2013). Damit der Forschungsprozess dem Kriterium der Offenheit gerecht wird, wurde während der Auswertung zudem eine induktive Kategorienbildung mittels der Zusammenfassenden Inhaltsanalyse zugelassen. In Anlehnung an Mayring (2010) wurde auf eine weitergehende hermeneutische Objektivierung verzichtet, insofern keine Ankerbeispiele benannt. Insgesamt liegt der Fokus auf der Strukturierten Inhaltsanalyse, im Speziellen auf der inhaltlichen Strukturierung mit dem Ziel „bestimmte Themen, Inhalte, Aspekte aus dem Material herauszufiltern und zusammenzufassen“ (Mayring 2010, S. 98).

Die Auswertung erfolgte unter Verwendung der Software MAXQDA. Insbesondere entwickelt für die Strukturierte Inhaltsanalyse ermöglicht das Programm die Definition und Verwendung von Codes und Subcodes, welche die Kategorien widerspiegeln, in geordneter Weise (Kuckartz 2010). Mittels einer methodisch kon- 
trollierten Verdichtung des Materials konnten fallübergreifende Aussagen zu Regelmäßigkeiten im Sinne der Forschungsfrage herausgearbeitet werden (ebd.), wobei durch die Nutzung von MAXQDA der Rückbezug zum Originaltext an jeder Stelle und $\mathrm{zu}$ jeder Zeit, schnell und einfach herzustellen ist.

Mit der Aufbereitung des theoretischen Vorverständnisses und dem exakten Einhalten des Forschungsablaufs wurde eine Transparenz geschaffen, die es ermöglicht, das Gütekriterium ,Verfahrensdokumentation` zu erfüllen. In der Interpretation werden die Ergebnisse in begründeter Form aufgearbeitet und gedeutet. Ebenso wie die Interpretationen muss die Angemessenheit der Kategoriendefinitionen der Überprüfung am Material standhalten. Diese wurden im Sinne der Intercoder-Reliabilität durch mehrere Forscher durchgeführt, wobei im gemeinsamen Forschungsdiskurs der Autoren Kategorien entstanden und zugeordnete Paraphrasen Dritten zur Überprüfung und Rückmeldung vorgelegt worden. Dabei handelte es sich sowohl um Forscher, die dem Thema nahestehen, wie auch um unabhängige Personen.

Die Rückmeldung der Beforschten wurde zum Zeitpunkt der Erhebung nicht eingeholt, insofern hat während des Forschungsprozesses keine kommunikative Validierung stattgefunden. Dennoch wurde durch die theoretische Vorarbeit und die Erstellung eines Leitfadens und letztlich auch durch die gezielte Wahl der Erhebungsmethode des problemzentrierten Interviews die Nähe zum Gegenstand gewahrt.

\section{Empirische Befunde aus der Fördermaßnahme „Nachhaltiges Landmanagement ${ }^{\text {"6 }}$}

In der nachfolgenden Interpretation sollen die aus den Interviews abgeleiteten und mittels der strukturierten Inhaltsanalyse reduziert erfassten Ergebnisse mit den theoretischen Ausführungen verknüpft werden, insofern handelt es sich um eine interpretative Verdichtung der Aussagen der Befragten Projektakteure. Sofern dabei Formulierungen aus der Befragung verwendet werden sind diese gekennzeichnet, ebenso die für die Interpretation genutzte Literatur. Die Autoren gehen von der Annahme aus, dass der Umgang mit neuartigem Wissen bei der Arbeit der Projekte im Nachhaltigen Landmanagement einen zentralen Stellenwert einnimmt. Die erforderliche „Nachhaltigkeit“ des Wissens kann nur dann entstehen, wenn das individuelle Wissen der einzelnen Projektteilnehmer kommuniziert und miteinander geteilt wird, somit ein kontinuierlicher wechselseitiger Wissenstransfer entsteht. Im Mittelpunkt des Forschungsinteresses steht die Frage, wie der kommunikative Austausch von Wissen intern und extern organisiert sein kann und muss, so dass die Teilnehmer über diese Kommunikation jeweils voneinander lernen. Mit einer verbesserten Einsicht in diesen Prozess wird sich der Umgang mit der Ressource Wissen im Projektverbund wirkungsvoller gestalten lassen. Transdisziplinarität wird als Ausgangsvoraussetzung verstanden. Ausgehend vom klassischen Verlauf eines Forschungsprozesses (vgl. APA 2012) kann festgestellt werden, dass spezifische Regeln oder Bedingungen des BMBF keine oder eine geringe präjudizierende Wirkung für die Wissensprozesse entfalten, die Befragten Akteure haben keine derartigen Hinweise gegeben. Die Ausprägungen sowie die Gestaltung von Kommunikation und Kooperation, des Lernens von und miteinander, werden deshalb als übertragbar auf andere Netzwerke oder 
Projektarbeiten angesehen, zumindest wenn unterschiedliche Akteure vor allem aus Wissenschaft und Praxis miteinander agieren. Trotzdem wirft die strukturelle verschiedenartige Anlage jedes Verbundprojektes die Frage auf, inwieweit die der nachfolgend beschriebenen Ergebnisse im Einzelfall übertragbar sind.

\subsection{Begriffsverständnis der Projektakteure}

Die Gestaltung der Transfer- und Implementierungstechniken ist in besonderem Maße von dem Verständnis der Begrifflichkeiten abhängig. Die Interviewpartner wurden befragt, welche Definition sie bei Transfer und Implementierung zu Grunde legen. In der inhaltsanalytischen Reduktion der Antworten ergab sich für die beiden Begriffe je eine allgemeine Definition.

Transfer wird fallübergreifend als die Kommunikation zwischen Wissenschaft und Praxis verstanden. Diese bezieht sich dabei auf den gerichteten Transfer von Ergebnissen an Praxisakteure und Stakeholder. Im Vergleich zu der theoretischen Ausarbeitung der Begrifflichkeit ist hier eine Diskrepanz feststellbar. Verstehen die Autoren Austauschprozesse als wechselseitige Kommunikation zwischen Sender und Empfänger, betrachten die Befragten Transfer eher als einseitigen nutzerorientierten Wissenstransfer, d. h. vom Wissensanbieter zum -nachfrager gerichteten Austausch. Er dient für die Aufklärungsarbeit, um Veränderungen im Sinne von nachhaltigem Landmanagement zu bewirken. Die Akteure sind sich im Klaren darüber, dass dafür offene Kommunikationswege nötig sind. Das verdeutlicht, welche tragende Rolle der Kommunikation nicht nur theoretisch, sondern auch in der praktischen Anwendung zukommt. Der Transfer wird von den Interviewpartnern als unproblematisch angesehen. Sie erkennen aber, dass der Prozess allein nicht für den Projekterfolg ausreicht. Transfer ist für die Befragten lediglich die Methode der Implementierung. Diese wird definiert als die optimale Folge von gutem Transfer. Sie spiegelt die praktische Umsetzung und Anwendung des Transfers wider.

Die Befragten gehen weiter davon aus, dass durch eine breitenwirksame Informationsverteilung das Wissen verwertet wird. Eine Spezifikation der auf individueller Ebene stattfindenden Lernprozesse konnte aus den Interviews nicht abgeleitet werden. Dies ist insofern verwunderlich, als dass die der bewusst eingesetzten Kommu-

Tab. 2 Begriffsverständnis der Befragten Projektakteure von Transfer und Implementierung

\begin{tabular}{lll}
\hline Begriffsverständnis & \\
\hline Transfer & Definition & Einseitiger Austauschprozess über offene Kommunikationswege \\
& Nutzerorientierter Wissenstransfer vom Wissensanbieter zum nachfrager \\
& Zweck & Aufklärungsarbeit \\
& Ziel & Veränderungen im Sinne von nachhaltigem Land-management bewirken \\
& Status & Unproblematisch \\
Implementierung & Definition & Optimale Folge von Transfer \\
& & Anwendung der in den Projekten entwickelten Methoden und Produkte \\
& Zweck & Anwendungsbezogene Verwertung von Wissen \\
& Ziel & Umsetzung von produktspezifischen Konzepten, Strukturen und Pro- \\
& zessabläufen sowie neuer Ideen \\
& Status & Problematisch \\
\hline
\end{tabular}


nikations- bzw. Lehrmethodik auf Senderseite keine Wirkungs- oder Lernerwartung auf Rezipienten- bzw. Lernerseite gegenübersteht. Alternativ haben die Autoren versucht, die Aussagen der Befragten zu interpretieren, umso indirekt Zusammenhänge zu ermitteln. Jedoch kann ein theorienahes Begriffsverständnis von Implementierung mit Fokus auf eben solche Lernprozesse nicht gefunden werden. Aus den Äußerungen der Interviewpartner kann aber geschlussfolgert werden, dass diese unbewusst durch die Implementierung der Projektergebnisse bei der Zielgruppe einen Lernprozess initiieren. Insofern ist das theoretische Konstrukt der Implementierung also nur in latenter Form anzutreffen. Wie das folgende Zitat zeugt ist die Orientierung bei der Implementierung ohnehin eher ökonomisch ausgerichtet, die Projekte sehen den Prozess als anwendungsbezogene Verwertung ihres Wissens: „Also direkte Implementierung war zum einen dabei, dass wir mit unseren Praxispartnern neue Produkte entwickeln.“" (2.1; Z. 141-142)

Ein Lernen findet, wenn überhaupt, nur durch die bzw. in der Anwendung des Produktes, respektive durch Aufklärung über dieses statt. Die Umsetzung von produktspezifischen Konzepten, Strukturen und Prozessabläufen sowie neuer Ideen steht im Mittelpunkt von Implementierung. Auf Grund des innovativen Charakters der Ergebnisse wird Implementierung als problematisch angesehen. Ein Grund dafür könnte aus theoretischer Sicht die unzureichende Aufklärung über Hintergründe des thematischen Fundaments sein. Als Ziel und Schwerpunkt der gesamten Projektarbeit wird Implementierung als rahmengebender Hauptprozess gesehen. Dieser wird durch Anwendung der in den Projekten entwickelten Methoden und Produkte erreicht.

Der langfristige Charakter von Implementierung, also die Verstetigung in Form des Erwerbs von Handlungsroutinen und Veränderung mentaler Modelle, wird im Projektablauf nicht direkt durch strategische Maßnahmen unterstützt. Dennoch bewirken die über das Projektende hinausgehenden Aktivitäten eine nachhaltige Nutzung der landwirtschaftlichen Ressourcen und ein Umdenken bei der Zielgruppe im Sinne der Zielsetzungen.

\subsection{Wissensbezogene Strategien}

Wie die Projekte Wissensprozesse gestalten, soll nachfolgend anhand der in der Theorie ausgearbeiteten Handlungsfelder Kommunikation und Lernen nachgezeichnet werden. Die von den Projekten nach außen gerichteten Tätigkeiten und Strategien innerhalb dieser Handlungsfelder initiieren Entwicklungsprozesse bei den Zielgruppen. In Ergänzung zu diesen deduktiven Kategorien, wird auf die induktiv gefundene Kategorie der Reflexionsschleifen eingegangen. Sie umfasst die von Projekten eingesetzten Methoden, welche die Projektarbeit und -ergebnisse reflektieren und somit helfen, mentale Veränderungsprozesse innerhalb der Projekte einzuleiten.

\subsubsection{Kommunikation}

Die Strategien der Befragten in Bezug auf Kommunikation sind durch eine skeptische Haltung gegenüber der Verwendung digitaler Medien gekennzeichnet. Zwar wird die Kommunikation über Telefon oder E-Mail gestaltet, jedoch liegt der Fokus für die Wissensvermittlung eher auf dem persönlichen Austausch. Dies ist synonym 
Tab. 3 Ergebnisse in der Kategorie Kommunikation

\begin{tabular}{|c|c|}
\hline \multicolumn{2}{|l|}{ Kommunikation } \\
\hline \multirow[t]{3}{*}{ Grundidee } & Wechselseitige Kommunikation zwischen unterschiedlichen Akteuren, Disziplinen etc. \\
\hline & Vorwiegend Informationsweitergabe und -austausch \\
\hline & Focus liegt auf der Bringschuld des Senders \\
\hline \multirow[t]{2}{*}{ Zweck } & Öffentlichkeitsarbeit \\
\hline & Interner Wissensaustausch \\
\hline \multirow[t]{2}{*}{ Ziele } & Interesse wecken und Veränderungen bewirken, begünstigen \\
\hline & Projektinterne Entwicklung von handlungsrelevanten Ergebnissen \\
\hline \multirow{4}{*}{$\begin{array}{l}\text { Methoden } \\
\text { interne } \\
\text { Kommunikation }\end{array}$} & Kommunikationskonzepte für den Wissenschafts-Praxis-Diskurs \\
\hline & Face-to-face-Kontakt \\
\hline & Informeller, partizipativer Austausch \\
\hline & Wenig Austausch über digitale Wege \\
\hline \multirow{5}{*}{$\begin{array}{l}\text { Externe } \\
\text { Kommunikation }\end{array}$} & Informative, öffentlichkeitswirksame Kommunikation \\
\hline & Marketingstrategien \\
\hline & $\begin{array}{l}\text { Einbezug öffentlichkeitswirksamer Persönlichkeiten, (politischer) } \\
\text { Entscheidungsträger }\end{array}$ \\
\hline & Praxisnahe Demonstrationen \\
\hline & Weiterbildungs-, Informationsveranstaltungen \\
\hline \multirow{5}{*}{$\begin{array}{l}\text { Regeln ge- } \\
\text { lingender } \\
\text { Kommunikation }\end{array}$} & Herstellen einer gleichen Basis/Einheitlichkeit (,,corporate identity“) \\
\hline & Vertrauensvolle, wertschätzende Kultur \\
\hline & Fehlertoleranz \\
\hline & Objektive Kommunikation \\
\hline & $\begin{array}{l}\text { Aufbereitung der Informationen je nach Rezipient Menge, } \\
\text { Art und Weise der Informationsweitergabe } \\
\text { Sprach- und Wortwahl } \\
\text { Wahl der Kommunikationsmittel }\end{array}$ \\
\hline \multirow[t]{2}{*}{ Tools } & E-Mail \\
\hline & Telefon \\
\hline
\end{tabular}

zu früheren Befunden (vgl. Köhler und Schilde 2003). Als Grund für dieses Verhalten nennen die Befragten neben dem erhöhten zeitlichen Aufwand für die Einführung digitaler Medien auch die Problematik, dass nur über face-to-face Kommunikation Mimik und Gestik, aber auch die haptischen Reize einbezogen werden können. Die Kommunikationstheorie bestätigt dementsprechend, dass diese Kommunikation bei identischen Zeit- und Ortsbedingungen eine geeignete Strategie ist, jedoch bei großen Verbundprojekten die zeitliche und örtliche Distanz, die diesen eigen ist, über elektronische Hilfsmittel aufzuheben ist (Probst et al. 1998).

Aufgrund der kulturellen Prägung/Einstellung der Projektbeteiligten, die sich durch eine fehlende Offenheit gegenüber neuen Medien auszeichnet, wird dieses Potenzial allerdings nicht erkannt. Vielmehr stellt der benötigte Kontroll- und Koordinationsbedarf, der für den Einsatz neuer Medien notwendig wird, ein nicht zu überwindendes Hindernis dar. Die Projektbeteiligten verwenden vorzugsweise die Strategie des persönlichen Treffens oder wählen den direkten Kontakt zu den Praxispartnern vor Ort, um an Informationen zu gelangen bzw. Wissen zu vermitteln. Sie sehen diese Methode als besonders geeignet für die Klärung von Problemen, wobei die informelle, vertrauensvolle Ansprache es ermöglicht, an implizite Informationen der Zielgruppe zu gelangen und Schwierigkeiten unbürokratisch zu lösen. 
Jedoch birgt diese informelle schnelle Kommunikation mit ihren kurzen Wegen das Risiko, dass nicht alle relevanten Beteiligten gleichermaßen informiert werden. Insofern sollten formale Wege gesucht werden, die eine Dokumentation nach sich ziehen. Dies ist umso wichtiger da die Kommunikation nicht nur der Verteilung von Wissen im Sinne von Information dient, sondern auch ein Teil der Nutzung dieses gemeinsamen Wissens ist (Gergen 1995).

Die Hauptaufgabe der Kommunikation, öffentlichkeitswirksamen Transfer topdown gerichtet zu vollziehen, wird um Strategien der Verstetigung ergänzt, damit die Wissensweitergabe in Implementierungen münden kann. Obschon allgemein anerkannt ist dies unter Wissenschaftlern oft nicht ausreichend akzeptiert, führt auch zu einer oft als eigenwillig zu bewertendem Kommunikationsverhalten (vgl. F\&L 2015). Aufgabe der Kommunikation ist also in erster Linie im Sinne einer push-Strategie, Wissen und Informationen vom Wissensanbieter, den Projektakteuren, an die Zielgruppe, die Wissensnachfrager, weiterzugeben. Bei den Projekten wird dahingehend eine informative, öffentlichkeitswirksame Kommunikation eingesetzt, die Informationen über Projektergebnisse publik macht und Interesse für das Thema in der Bevölkerung weckt. Die Projekte entwickeln weiterhin anregende Kommunikationskonzepte für den Wissenschafts-Praxis-Diskurs, wobei sie selbst informative, für das Projekt nicht direkt nutzenrelevante Informationen, die sie kommunikativ vermittelt bekommen, für den Überblick nutzen. Für die externe Kommunikation werden Marketingstrategien eingesetzt, wobei ersichtlich wird, dass der Einbezug von öffentlich bekannten bzw. anerkannten Personen förderlich für das Publikmachen des Projektthemas ist. Für die interne Kommunikation, die Transparenz schaffen sollte, hat sich innerhalb der Projekte als Kommunikationsmittel die Berichtspflicht sowie face-to-face orientierte Statuskonferenzen bewährt.

Wichtig erscheint es für die Interviewpartner, die Kommunikation vorab konzeptuell mittels eines Kommunikationskonzeptes auszuarbeiten und zu planen. Die Interviews zeigen aber auch, dass nicht in allen der untersuchten Projekte darauf geachtet wird, dass insbesondere für die Praxispartner ein solches Konzept vorliegt. Dies steht im Widerspruch dazu, dass ein in der Projektplanung verankertes Kommunikationskonzept, in dem Strategien, Kanäle und Kommunikationsnetzwerke festgehalten werden, gewährleisten kann, dass der Wissensträger sein Wissen nicht nur im Team öffentlich macht (Zscheischler et al. 2012). Ebenso muss auf die Bedürfnisse der Wissensnachfrager mit adäquaten Kommunikationsmitteln reagiert werden. Dabei ermöglichen die in der Planung vorgeschriebenen Kommunikationswege eine effiziente und effektive Kommunikation. Jedoch wird in den Projekten die konzeptuelle Orientierung eher in latenter Form vollzogen. In den Interviews können verschiedene Regeln identifiziert werden, die für die kommunikativen Prozesse strategische Beachtung finden. So definieren die Befragten das Herstellen einer gleichen Basis und das Schaffen einer Einheitlichkeit im Sinne einer „corporate identity“ als Voraussetzung für eine gelingende Kommunikation: Auf der Ebene einer gemeinsamen vertrauensvollen, wertschätzenden Kultur stellen die Befragten fest, dass die Offenlegung von Schwächen des Themas sowie die Kommunikation konkret abgesicherter Daten positiv auf Praxispartner wirkt. Die Kommunikation wird daher im weitesten Sinne objektiv gestaltet. Das heißt, die angesprochene offene Atmosphäre sollte ausgebaut und eine Fehlertoleranz explizit zugelassen werden. 
Um eine Kommunikationsstrategie ergebnisreich gestalten zu können, plädieren die Interviewpartner dafür, diese am Kommunikationsbedarf der Akteure auszurichten. Informationen müssen also je nach Rezipient zielgruppengerecht oder wissenschaftlich aufbereitet sein. Sowohl die Menge wie auch Art und Weise der Informationsweitergabe sind zielgruppenabhängig, ebenso erfolgen die Sprach- und Wortwahl sowie die der Kommunikationsmittel in diesem Sinne.

Mit Blick auf die Reichweite wurde die Kommunikation meist bewusst breitenwirksam in alle Richtungen verlaufend eingesetzt, so dass, neben einer direkten Ansprache und Einbezug der Praxispartner, auch ein wechselseitiger Austausch ermöglicht wird. Bewährt haben sich dabei einerseits die top-down, andererseits aber auch die bottom-upKommunikation. Letztere umfasst die Holschuld der Empfänger. Der Empfänger wird zwar im Sinne der push-Strategie über ansprechende Informationen angeregt, muss aber in der Konsequenz selbst aktiv werden und im Zuge seiner Holschuld und des pull-Prinzips Wissen einfordern (Herbst 2000). Damit dies gelingt, werden diese Personen kommunikativ vernetzt. Als wichtig erachten die Befragten dabei einen regelmäßigen Austausch bei dem auf die Bedürfnisse der Kommunikationspartner eingegangen wird. Dabei entscheidend ist, dass die Projektpartner Interesse an der Zielgruppe zeigen sowie ansprechend, aktivierend, flexibel und anpassungsfähig auf Rezipienten zugehen. Nur so kann auf ein Interesse gestoBen werden, sind sich die Befragten einig.

Grundsätzlich liegt bei den Projekten eine Bringschuld des Wissensanbieters vor. Die Gesprächspartner äußern vor allem, dass die kommunikative Strategie vom Sender ausgehen muss, d. h. dieser direkt zu den Praxispartnern vor Ort oder den Projektpartnern geht und diese informiert. Für den Gesprächseinstieg hat sich die Strategie des SmallTalk als besonders effektiv herausgestellt. Die Einrichtung praxisnaher Demonstrationen, um Projektergebnisse zu kommunizieren, erwies sich ebenfalls als zweckdienlich für die Wissensverteilung an die Zielgruppe. In Form von face-toface Meetings und geplanten Treffen zum Austausch mit den Projektebeteiligten wird Kommunikation strategisch zielgruppengerecht gestaltet.

Bei der Sender-Kommunikation geht es in Hinblick auf Zielgruppe und Praxis insbesondere um die Erzeugung von Aufmerksamkeit für das Projekt. Dies ist aber nicht im Sinne eines Marketings gemeint, sondern spiegelt die Offenheit für externe Fragen wider, um so Praxisakteure für das Projektthema zu begeistern. Gestaltet wird dies durch den Einbezug der Zielgruppe bzw. der Vernetzung der Akteure mithilfe der Kommunikation. Ein geplantes Zusammenbringen von Kommunikationspartnern auch über das Projekt hinaus ist hierbei besonders effektiv. Mittels diesen gezieltem Treffen von Akteuren unterschiedlicher Disziplinen wird ein Austausch und Kontakt angeregt. An dieser Stelle wirkt sich auch die Kommunikation mit Entscheidungsinstanzen förderlich auf die Projektarbeit aus. Nach außen publik gemacht wird das Projekt vor allem über die Presse, wobei auch eine breitenwirksame Kommunikation auf diversen Veranstaltungen erzielt wird. Die gerichtete Kommunikation, vom Sender ausgehend, wird in allen Projekten durch den Fokus auf einen wechselseitigen Austausch ergänzt. Dieser wird von den Befragten auf allen Ebenen durchgeführt. Angefangen von der Wechselseitigkeit innerhalb einer Projektgruppe, d. h. Wissenschaftler, Arbeitsgruppe und/oder Teilgruppe, über einen Austausch mit Produktherstellern, Zielgruppe oder Externen, bis hin zu dem Einbezug bzw. der 
Kommunikation mit politischen Entscheidungsträgern. Die Befragten achten darauf, dass ausgewählte Kommunikationspartner förderlich für das Projekt sind. Aus diesem Grund wird beispielsweise bei Problemen mit externen qualifizierten Akteuren gesprochen, um Lösungen zu finden. Mit politischen Entscheidungsträgern wird in den wechselseitigen Diskurs getreten, um Rahmenbedingungen, die das Projekt hindern, zu verändern. Als Kommunikationsmittel werden auch hier insbesondere Gespräche im Sinne von Treffen vor Ort, Messen, Konferenzen oder Diskussionsforen arrangiert. Aber auch gezielte Interviews bzw. Befragungen oder Weiterbildungsveranstaltungen eignen sich, um in den Austausch mit Rezipienten zu kommen. Ein direkter, persönlicher und partizipativer face-to-face-Austausch wird von den Interviewpartnern fast ausschließlich ausgewählt. So können die Projektbeteiligten auch spontan und ungezwungen in einen Diskurs mit Akteuren oder potenziellen Umsetzern treten. Die wechselseitige Kommunikationsstrategie eignet sich für verschiedene Aspekte der Projektarbeit. Innerhalb des Projektes wird sie angewandt für eine gemeinsame Entwicklung von handlungsrelevanten Ergebnissen, für die Erstellung von Messinstrumenten, für Reflexionsschleifen oder für den Austausch unterschiedlicher Sichtweisen. Gerade im Hinblick auf die Herausforderungen, die der transdisziplinäre Charakter der Projekte mit sich bringt, ist ein partizipativer Austausch anzustreben. Dies sorgt für eine Wertschätzung der Projektpartner, um gemeinsam darauf aufbauend einheitliche Botschaften zu entwickeln.

Wie sich zeigt, setzen die Befragten vielfältige Strategien ein, um entsprechend dem Gegenüber Kommunikationskanäle und -orte auszuwählen. Der Einsatz unterschiedlichster Mittel erfolgt dabei, um nicht nur die Zusammenarbeit zu gestalten, sondern auch um die Öffentlichkeit zu erreichen. Die Interviewpartner achten auf eine einheitliche Mittelwahl, welche reflexive Prozesse fördert. Wie bereits zu Beginn beschrieben, sind sich die Befragten zwar einig darüber, dass digitale Kommunikationskanäle schnell, informativ und breitenwirksam sind, jedoch wählen sie diese vor allem als Informationskanäle, weniger als Implementierungsmittel. Zu den digitalen informativen Transfermitteln zählen konkret E-Mail und Telefon, die als Kommunikationskanäle Zeit sparen und eine bessere Erreichbarkeit ermöglichen.

Kommunikationskanäle werden weiterhin für den fachlichen Austausch und den Vertrauensaufbau und Wertschätzung sowie im Allgemeinen für den Wissensaustausch als wichtig angesehen. Für die Kommunikation in die Praxis empfehlen die Befragten den Einsatz anregender und insbesondere für Praktiker aktivierende Kommunikationskanäle.

In allen Fällen geht es um den wechselseitigen Austausch zwischen Wissensanbieter und -nachfrager. Insofern lässt sich kein direkter Bezug zum Paradigma der Massenkommunikation herstellen. Für die Projektinterne wie auch -externe Kommunikation kann es zudem spezifische Abweichungen geben. Als analoge Transfermittel mit kommunikativem Charakter können vorwiegend face-to-face-Gespräche mit Akteuren oder aktivierende Befragungen identifiziert werden. Digitale Transfermittel werden eher für die projektinterne Kommunikation eingesetzt. Dabei handelt es sich insbesondere um E-Mail, Telefon oder interne Homepage-Bereiche als Mittel für den projektinternen Austausch. Nur in einzelnen Fällen werden ein Projektmanagementtool, Skype, Social Media oder Diskussionsforen genutzt, zudem meist nur ansatzweise. Auch Veranstaltungen werden als kommunikative Treffen ausgewählt, auf 
denen Transfer stattfindet. Workshops, Messeauftritte, Feldtage, Bürgerforen oder ein Runder Tisch sind Mittel für eine Außenkommunikation, welche von den Projektakteuren angegeben werden. Allgemein lag der Fokus beim persönlichen Treffen auf unterschiedlichsten Ebenen, wobei für den internen Austausch insbesondere Tagungen, Konferenzen oder Diskussionsforen genutzt bzw. eigens veranstaltet wurden.

Wissensbestände können sich bei den Projektakteuren nur dann entwickeln, wenn Informationen in geeigneter Form zur Verfügung gestellt werden. In einem weiteren Schritt kann dieses Wissen dann kombiniert und letztlich in Handlungen umgesetzt werden. Aus theoretischer Sicht ist dabei zu beachten, dass explizite Wissensinhalte in mündlicher Form dynamisch und unstabil sind, in gespeicherter Form, sei dies analog oder digital, statisch und stabil und somit für ein breites Publikum nutzbar (Hasler Roumois 2010). Der Fokus der Projekte liegt offenkundig auf dem externen, informellen Transfer. Da die Projekte sich meist in der Abschlussphase ihres Projektzyklus befinden ist dies nachvollziehbar. Insofern geht es den handelnden Projektakteuren, die hier interviewt wurden, jetzt vorrangig um die außenwirksame Präsentation ihrer Ergebnisse. Dennoch sind interne Wissensaustauschprozesse - bspw. mit dem Ziel der Projektdokumentation und der Nachhaltigkeit - ebenso wichtig und salient zu gestalten wie anschließende öffentlichkeitswirksame Verbreitungsstrategien.

\subsubsection{Lernen}

Bei der Strategie des Lernens liegt der Fokus der Projekte auf den Lernprozessen der Zielgruppe. Die Ergebnisse aus den Interviews werden in Tab. 4 kurz zusammengefasst.

Die Maßnahmen richten sich nach außen an projekt-externe Wissensnachfrager. Damit eine Umsetzung des generierten Wissens stattfindet, müssen neben Handlungen auch Kompetenzen entstehen. Grundlage dafür sind Internalisierungsprozesse des Wissens. Insofern ist es folgerichtig, dass die Projekte versuchen über die Gestaltung einer ansprechenden Lernumgebung die Wissensnutzung zu unterstützen. Lernen als Inbegriff von Implementierung sollte in einer wissensbasierten Projektarbeit im Mittelpunkt der strategischen Ausrichtung stehen. Die bloße Bereitstellung von Lernangeboten ist jedoch eher mit einer nicht nachvollziehbaren Entstehung

Tab. 4 Ergebnisse in der Kategorie Lernen

\begin{tabular}{ll}
\hline Lernen & \\
\hline Grundidee & Maßnahmen richten sich nach außen an projekt-externe Wissensnachfrager \\
& Ansprechende Gestaltung der Lernumgebung \\
& Praktische Erfahrungen ermöglichen \\
& Lernen am Objekt \\
& Lernprozessen bei der Zielgruppe initiieren \\
Zweck & Unterstützung der Wissensnutzung \\
& Umsetzung des generierten Wissens \\
Ziel & Evaluationen \\
Methoden & Feldtage \\
& Workshops, Schulungen, Weiterbildungen \\
& Lernmittel (Bücher, Curricula, Merkblätter) \\
\hline
\end{tabular}


von Wissen bei den eventuellen Nutzern diese Lernangebote verbunden. Damit dies planmäßig geschieht ist eine Steuerung erforderlich, müssen individuelle und kollektive Wissensbasen systematisch zusammengebracht und Lernprozesse angeregt werden (vgl. u. a. Hacker et al. 2011). Lernen bedeutet, explizites Wissen, welches durch Information, Dokumentation und Kommunikation bereitgestellt wird im Zuge der Internalisierung zu verinnerlichen, mit dem Ziel eine Verhaltensänderung zu bewirken.

Der strategische Einsatz des Lernens für Transfer und Implementierung heißt für die Befragten, dass Instrumente wie beispielsweise Evaluationen eingesetzt werden, damit die Projektakteure durch Reflexion der Projekterkenntnisse Schlussfolgerungen ziehen, ihre Handlungsweisen im Projekt anpassen können. Außerdem planten die Interviewpartner verschiedene Veranstaltungen für unterschiedliche Zielgruppen, damit sie nicht nur Informationen transferieren, sondern zudem eine Implementierung von Wissen, d. h. ein Lernen bei dieser Gruppe initiieren können. Die Informationen werden anschaulich anhand konkreter Handlungsbeispiele: „Wir haben konkret Workshops gemacht. Und wir haben einen Feld-Tag gemacht. Ursprünglich/. Ich wollte eigentlich 2 Feld-Tage machen. Der Feld-Tag hat sehr sehr gut funktioniert. Das war richtig knacken voll.“ (2.5; Z. 1357-1360)

Im Sinne des Lernens am Objekt setzen die Gesprächspartner so eine zielgruppenadäquate Lernstrategie ein. Ausgerichtet wird das Lernen direkt in der Praxis. In diesen Lernarrangements wird es den Rezipienten ermöglicht eigne praktische Erfahrungen zu machen. Diese Freiheiten erlauben nicht nur Selbstlernprozesse, sondern insbesondere auch ein voneinander Lernen in der Akteurgruppe. Unterstützt wird dies von den Projektpartnern, indem sie die zu vermittelnden Inhalte stetig wiederholen. Konkrete Anhaltspunkte, wie das Lernen gestaltet wurde bzw. von statten ging, finden sich in den Interviews nicht. Obschon nach Verhaltensänderung, Wissenserwerb und Bewusstseinswandel gefragt wurde steht das Lernen nicht im Vordergrund und wird nur vereinzelt thematisiert wird. Bei den Projektakteuren passieren diese Prozesse eher unbewusst, ihre Entwicklungs- und Forschungsprojekte haben nicht zwangsläufig die Initiierung von Lernprozessen zum Ziel und evtl. Maßnahmen laufen auch nicht unter diesem Label. Dennoch werden als konkrete Implementierungsmittel von Wissen direkte und thematische Lernveranstaltungen wie Schulungen, Workshops, Weiterbildungen sowie Lernmittel wie Bücher, Curricula oder Merkblätter organisiert. Entwickelte Lernkurse oder spezielle Feldtage helfen außerdem, effektiv Lernen zu bewirken.

Es zeigt sich, dass die Projektakteure Lernstrategien einsetzten, die im Abgleich mit der Theorie, als hochwirksam und außerordentlich zielgruppengerecht angesehen werden können. Über die Praxisnähe wird der Sinnbezug hergestellt, durch die Anwendung werden haptische Reize angesprochen, so dass eine Internalisierung und Konstruktion des Wissens beim Rezipienten stattfinden kann (vgl. Nolda 2008). Wenn zukünftig mehr als nur punktuelle Lernaktivitäten etabliert und umgesetzt werden sollen, wären diese Aspekte zukünftig noch stärker zu berücksichtigen. Mit dem gesetzten Ziel der Implementierung scheint dies nur folgerichtig, da es nicht nur Handlungen anzuregen gilt, sondern im Sinne einer Verstetigung Kompetenzen und somit die Entwicklung von, im Falle des nachhaltigen Landmanagements, pränormative Vorstellungen anzuregen. 


\subsubsection{Reflexionsschleifen}

Bezogen auf die Projekte selbst konnten reflexive Lernprozesse erhoben werden, die in die Kategorie ,Reflexionsschleifen' eingeordnet wurden. Hierunter werden Maßnahmen gefasst, die dem internen Lernen dienen. In den Interviews zeigt sich, dass die Projektbeteiligten aus Ereignissen bzw. Erfahrungen Rückschlüsse für ihr weiteres Vorgehen ziehen. Diese sogenannten Reflexionsschleifen bewirken Änderungen in unterschiedlicher Hinsicht, die Mehrzahl bezieht sich aber auf die Modifikation der Umgangsformen.

Projektübergreifend herrscht die Meinung, Kommunikationsformen müssen mit der jeweiligen Zielgruppe bzw. deren Bedürfnisse in Einklang gebracht werden. Das bestätigt sich in den Aussagen über den Mitteleinsatz und knüpft an die Kommunikationskanäle, die je nach Adressat abgestimmt werden, sowie an die Anpassung der Sprache je nach Reaktion der Rezipienten an. Darauf aufbauend zeigt sich, dass die Forschung an eben genau diese Zielgruppenbedürfnisse angepasst werden muss, um eine Umsetzung zu garantieren. So können sich Folgeprojekte an den aktuellen Projektproblemen orientieren, wobei eine Konzeptanpassung für eine Übertragbarkeit vorgenommen werden muss. Die Instrumente oder Tools der Reflexion werden je nach Projekt unterschiedlich eingesetzt. So wurde in einem Projekt eine SWOT-Analyse in der Mitte der Projektlaufzeit realisiert, welche die Reflexion der Projektrealität erlaubte. Bei dieser internen Evaluation war zudem eingeplant, Anpassungen der Kooperation durchzuführen, falls entscheidende Probleme bei der Zusammenarbeit aufgedeckt worden wären.

Als wichtig für Reflexionsmöglichkeiten werden neben dem expliziten Einbezug von Praxisrückmeldungen auch Instrumente für den Erhalt eines Überblicks als wichtig befunden. Letzteres wird in einem Projekt durch einen nachträglich installierten Controlling-Bericht gestaltet, um Informationen aus anderen Teilprojekten zu bekommen. Weiterhin erkannten die Interviewten, Evaluationen am Projektende mit allen Teilnehmern seien notwendig. Dies würde die Möglichkeit bieten, umfassend über das Projekt zu reflektieren. Außerdem müssen Reflexionsinstrumente für den Einbezug der Zielgruppe installiert werden. Dabei ist es notwendig Rückmeldungen von der Zielgruppe explizit einzufordern.

In den Interviews kristallisieren sich letztendlich drei Reflexionsthemen heraus. Einmal wird über Instrumente reflektiert. Des Weiteren über die Projektrealität, um Problemen zeitnah entgegensteuern zu können. Und ebenfalls über den Projektablauf, um eine Weiterarbeit planen zu können.

\section{Fazit: Wissenskommunikation versus Organisation gemeinsamen Lernens}

\subsection{Zusammenfassung}

Aus den Interviews mit den Koordinatoren und Praxisakteuren konnten entsprechend der Forschungsfrage, wie Projektakteure Transfer- und Implementierungsprozesse von Wissen im Projektverbund gestalten, zahlreiche Erkenntnisse herausgearbeitet werden. Im ersten Schritt wurden fallübergreifend für alle Projekte ähnliche Begriffs- 
verständnisse eruiert. Transfer sehen diese als (außenwirksame) Kommunikation zur Zielgruppe über verschiedene analoge und digitale Mittel. Implementierung ist für sie die Umsetzung der aus dem Projekt transferierten Wissensinhalte durch den Nutzer. Im Vergleich mit den theoretisch der Arbeit zugrunde liegenden Definitionen lässt sich eine Schnittmenge bezüglich Kommunikation und Umsetzung feststellen. Der Aspekt des Lernens, welcher bei den Autoren als Implementierungsmethode gilt, wurde in den Interviews nur punktuell explizit betont.

Da sich die Projekte eher am Ende ihres Entwicklungszyklus befanden liegt der Schwerpunkt ihrer Strategien vornehmlich auf den Transferprozessen. Eine Implementierung wird dennoch als Ziel der Projekte angestrebt. Als Instrumente konnten verschiedene Mittel herausgearbeitet werden, die strategisch für Transfer und Implementierung eingesetzt werden, wobei die Projekte diese Mittel universell einsetzen. Vor allem digitale Medien wie Homepage, Newsletter oder explizit für den internen Transfer Speicherorte wie Dropbox werden verwendet. In Widerspruch zu diesem Einsatz sogenannter ,Neuer Medien', steht die skeptische Einstellung aller Befragten zu diesen Transfermitteln. Sie benennen gleichwohl die Vorteile digitaler Instrumente wie Zeitersparnis, Ortsunabhängigkeit und finanzielle Ersparnisse. Dennoch sehen sie nicht die Notwendigkeit der Einführung einer technologiebasierten Austauschplattform!

Für den externen Transfer setzen die Befragten vor allem auf informelle Austauschprozesse mit den Projektpartnern. Insbesondere in Bezug auf die Praxispartner ist dies eine bewährte strategische Vorgehensweise. Über den informellen Weg werden vorzugsweise bestehende Beziehungen in die Arbeit eingebunden. Im gemeinsamen Diskurs werden die vorhandenen Wissensbestände der Partner ausgetauscht, genutzt und ergänzt, das neu geschaffene Wissen weitergetragen.

Die Außenkommunikation funktioniert in den Projekten gut. Trotz eines unidirektionalen Transferverständnisses werden auch reziproke Kommunikationsprozesse betont. Aus den Interviews geht hervor, dass die Akteure die Strategie verfolgen, durch das Beachten verschiedener Regeln einen optimalen Transfer mit anschließender Implementierung zu erreichen. Die Regeln erleichtern die Austauschprozesse hin zu den Wissensnutzern. Die Projektakteure als Sender agieren dahingehend zielgruppenspezifisch und zielgruppennah. Im partizipativen Prozess versuchen sie die Transdisziplinarität strategisch zu lösen in dem sie eine gemeinsame Kultur herstellen und adäquate Mittel einsetzen. Sie setzen auf Wechselseitigkeit und reflexive Prozesse, die innerhalb des Projektes zur Anpassung von Methoden, Instrumenten und Vorgehen führen. Der Implementierungsgedanke ist dabei stets salient. Auch diesbezüglich wenden die Interviewpartner spezielle Strategien an. Es sind die gleichen Mittel wie beim Transfer, die zum Einsatz kommen. Informative Veranstaltungen zählen ebenso dazu wie digitale Medien. Der Fokus liegt auf einer breitenwirksamen Präsentation. Zielgruppenspezifisch - wenn auch das Lernen nur latent bei den Projektakteuren ist - schaffen diese eine lernförderliche Umgebung. Durch besonders praxisnahe Veranstaltungen wird das Lernen am Objekt gefördert. Das Vorstellen von beispielhaften Umsetzungen hat sich als besonders wirksame Methode erwiesen.

Damit die Strategien weiter ausgebaut werden können, ist es ratsam, im Sinne eines Wissensmanagements, die Prozesse der Kommunikation und des Lernens bewusst zu machen sowie über die punktuelle Gestaltung hinaus Kommunikation 
und Lernen strategisch zu konzeptionieren. Dazu zählt, dass nicht nur die Erfahrungen inhaltlicher Natur ausgetauscht werden. Es gilt gleichsam Erfahrungen konsequent zu explizieren und auszutauschen, die die Projektarbeit betreffen. Sind diese in formeller, d. h. schriftlich dokumentierter Form abgelegt, können nachfolgende Projekte darauf aufbauen und Folgeprojekte von den gemachten Erfahrungen profitieren. Dazu ist es förderlich, wenn auf eine fehlertolerante Kultur zurückgegriffen werden kann. Denn erst durch das Zulassen von Fehlern kann sich eine lernfreundliche Atmosphäre bilden, die es zulässt, aus einem Irrtum einen positiven Rückschluss auf die weitere Projektarbeit zu ziehen. Am Ende, wenn es ermöglicht wird interne und externe Transfer- und Implementierungsstrategien sowohl auf die inhaltliche Vermittlung als auch auf die Vermittlung von Projektprozessen anzuwenden, kann sich - im Sinne einer Organisationsentwicklung - eine wissensbasierte Projektarbeit etablieren, in der das Konzept eines Wissensmanagements einfließen kann. Die Auswertung der Interviews hat gezeigt, dass zahlreiche gute Ansätze vorhanden sind, die einen profitablen Ausbau ermöglichen. Besondere Beachtung verdient hierbei die nicht immer in ihren Potenzialen ausgeschöpfte inter- und transdisziplinäre Zusammenarbeit sowie die erarbeiteten Erkenntnisse zum Erfolg unterschiedlicher Wege.

\subsection{Bewertung der Untersuchung}

$\mathrm{Da}$ in der vorliegenden Untersuchung auf Projekte einer BMBF-geförderten Maßnahme Bezug genommen wird, muss der Transfer der Ergebnisse auf nicht-forschungsspezifische Gruppen hinterfragt werden. Spezifische Auswirkungen der Strukturen eines Forschungsverbundes konnten in der Erhebung nicht ausgemacht werden. Die einzelnen Projekte werden als in sich geschlossene Netzwerke verstanden, die langfristig eine auf Freiwilligkeit beruhende Kooperationsstruktur zum Ziel haben, da sie, wie andere Projekte und Gruppenvorhaben auch, zeitlich beschränkt sind. Aus diesem Grund sind der Wissenstransfer und die Implementierung zentrale Aspekte, die von den Beteiligten verfolgt werden, um ihre Ergebnisse nachhaltig zu sichern. Die Strategien und letztlich die verwendeten Tools können also auch Hinweise auf Kommunikations- und Kooperationsstrukturen außerhalb von Forschungsverbünden geben und somit auf Projekte unterschiedlichsten Inhaltes übertragen werden. Dabei können die Angaben der befragten Projektakteure als vergleichsweise gesichert und weniger als sozial erwünscht angenommen werden, da die Interviews von einem unabhängigen Forscherteam durchgeführt wurden. Der Auftraggeber in Form des wissenschaftlichen Begleitvorhabens war als solches bekannt, wobei darauf verwiesen wurde, dass die Erhebung der wissenschaftlichen Expertise gilt und keine negativen Konsequenzen für die Projekte zu erwarten sind. Nicht zuletzt, da die Interviews in anonymisierter Form ausgewertet wurden.

Aus der Untersuchung kann geschlossen werden, dass Transfer und Implementierung nicht zwangsläufig abhängig von bestimmten übergeordneten Strukturen, sondern eher als allgemeine, aber zwingend notwendige Prozesse zu verstehen sind - deren Gestaltung erfolgsversprechend für die langfristige Wirkung von Projekten und deren Ergebnisse ist. 


\section{Anmerkungen}

1 Das Modul A wird im fortlaufenden Aufsatz nicht weiter betrachtet, da es nicht Forschungsgegenstand der Untersuchung war. Dennoch sei an dieser Stelle erwähnt, dass in Bezug auf Kommunikation und Kooperation, insbesondere aber bezüglich des Transfers und der Implementierung von in den Projekten gewonnen Wissen, strukturell und methodisch ähnliche Ansätze wie in Modul B zu finden sind.

Open Access Dieser Artikel unterliegt den Bedingungen der Creative Commons Attribution License. Dadurch sind die Nutzung, Verteilung und Reproduktion erlaubt, sofern der/die Originalautor/en und die Quelle angegeben sind.

\section{Literatur}

APA. (2012). Publication Manual of the American Psychological Association (6. Aufl.). Washington: APA Press.

Baum, H., Freitag, M., Köhler, T., \& Schilde, P. (2003). Organizational barriers of inter-firm cooperation and how to overcome them: The two-level cooperation model. In I. Johansson (Hrsg.), Entrepreneurship, spatial industrial clusters and inter-firm networks. Udevalla: Proceedings of the Uddevalla Symposium.

BMBF Bundesministerium für Bildung und Forschung. (2013). Nachhaltiges Landmanagement. Broschüre. Bonn: BMBF.

Camargo-Borges, C., \& Rasera, E. F. (2013). Social constructionism in the context of organization development dialogue, imagination, and co-creation as resources of change. SAGE Open. doi:10.1177/2158244013487540. (Published 3 May 2013)

Davenport, T. H., \& Prusak, L. (1998). Wenn ihr Unternehmen wüsste was es weiß ... Das Praxishandbuch zum Wissensmanagement. Landsberg: Verlag Moderne Industrie.

F\&L. (2015). Social Media in der Wissenschaft; In: Forschung und Lehre, Heft 08/2015. http://www. forschung-und-lehre.de/wordpress/Archiv/2015/ful 08-2015.pdf.

Freeman, L. C. (2006). The development of social network analysis. Vancouver: Empirical Press.

Gergen, K. J. (1995). Social construction and the educational process. In L. P. Steffe \& J. Gayle (Hrsg.), Constructivism in education. Hillsdale: LEA.

Gergen, K. J. (2009). Relational being - beyond self and community. Cambridge: Harvard University Press.

Hacker, W., Pietrzyk, U., \& Debritz, U. (2011). Wissen erfolgreich weitergeben. Lösungen für kleine und mittlere Unternehmen im demographischen Wandel. Lengerich: Pabst Science Publishers.

Härtel, L., \& Hoffmann, M. (2013). Transfer- und Implementierungsstrategien von Wissen im Projektverbund. Empirische Untersuchung am Beispiel der BMBF Fördermaßnahme ,Nachhaltiges Landmanagement; Forschungsbericht in Form einer Masterarbeit, TU Dresden: unveröffentlicht.

Hasler Roumois, U. (2010). Studienbuch Wissensmanagement. Zürich: Orell Füssli Verlag AG.

Herbst, D. (2000). Erfolgsfaktor Wissensmanagement. Berlin: Cornelesen Verlag.

Hiltz, S. R., \& Turoff, M. (1993). The network nation: Human communication via computer. London: MIT Press.

Janse, G. (2007). Communication between forest scientists and forest policy makers in Europe - A survey on both sides of the science/policy interface. Forest Policy and Economics, 10(2008), 183-194.

Johnson, J. D., \& Chang, H.-J. (2000). Internal and external communication, boundary spanning, and innovation adoption: An over-time comparison of three explanations of internal and external innovation communication in a new organizational form. Journal of Business Communication, 37(3), 238-263.

Kaiser, D. B., Köhler, T., Weith, T., Gaasch, N., Rogga, S., Strauß, C., \& Zscheischler, J. (2012). Informations- und Wissensmanagement im Nachhaltigen Landmanagement: Konzeptionelle Grundlagen für die umsetzungsorientierte Forschung [Elektronische Ressource]. Müncheberg: Leibniz-Zentrum für Agrarlandschaftsforschung (ZALF).

Kaiser, D. B., Weith, T., \& Köhler, T. (2015). Knowledge management in sustainability research projects - concepts, effective models and examples in a multistakeholder environment. Journal of Applied Environmental Education \& Communication. (in press) 
Knieling, J., \& Müller, B. (Hrsg.). (2015). Klimaanpassung in der Stadt- und Regionalentwicklung, Ansätze, Instrumente, Maßnahmen und Beispiele, Reihe Klimawandel in Regionen zukunftsfähig gestalten (Bd. 7). München: oekom-Verlag.

Knieling, J., \& Roßnagel, A. (Hrsg.). (2015). Governance der Klimaanpassung. Akteure, Organisation und Instrumente für Stadt und Region, Reihe Klimawandel in Regionen zukunftsfähig gestalten (Bd. 6). München: oekom-Verlag.

Köhler, T., \& Schilde, P. (2003). From project teams to a virtual organization: The case of the education portal Thuringia. Frontiers of e-Business Research, 2(2).

Kreitel, W. A. (2008). Ressource Wissen. Wissensbasiertes Projektmanagment erflogreich im Unternehmen einführen und nutzen. Wiesbaden: Gabler.

Kuckartz, U. (2010). Einführung in die computergestützte Analyse qualitativer Daten. Wiesbaden: VS Verlag Sozialwissenschaften.

Küster, H. (2012). Die Entdeckung der Landschaft. Einführung in eine neue Wissenschaft. München: C.H. Beck. (Beck'sche Reihe 6061).

Larsen-Freeman, D. (2013). Language learning. Supplement, 63.

Lave, J., \& Wenger, E. (1991). Situated Learning: Legitimate Peripheral Participation. Cambridge: Cambridge University Press.

Mayring, P. (2010). Qualitative Inhaltsanalyse. Grundlagen und Techniken. Weinheim: Beltz Verlag.

Nolda, S. (2008). Einführung in die Theorie der Erwachsenenbildung. Darmstadt: WGB.

North, K., Romhardt, G., \& Probst, K. (2000). Wissensgemeinschaften. Keimzellen lebendigen Wissensmanagements. In: IO-Management, 7(7), 52-62.

Oberschulte, H. (1996). Organisatorische Intelligenz - ein Vorschlag zur Konzeptdifferenzierung. Berlin: Walter de Gruyter \& Co.

Probst, F., Raub, S., \& Romhardt, K. (1998). Wissen managen. Wie Unternehmen ihre wertvollste Ressource optimal nutzen. Wiesbaden: GWV Fachverlage.

Prokesch, S. E. (1997). Unleashing the power of learning: A interview with British Petroleum's John Browne. Harvard Business Review, 75(5), 147-168.

Reid, R. S., Nkedianyea, D., Saida, M. Y., Kaeloa, D., Nesellea, M., Makuia, O., Onetua, L., Kiruswad, S., Ole Kamuaroa, N., Kristjansona, P., Ogutua, J., BurnSilverh, S. B., Goldmani, M. J., Boonec, R. B., Galvinc, K. A., Dicksonj, N. M., \& Clarkj, W. C. (2009). Evolution of models to support community and policy action with science: Balancing pastoral livelihoods and wildlife conservation in savannas of East Africa. In: PNAS Early Edition, S. 1-6. www.pnas.org/cgi/doi10.1073/pnas.0900313106.

Rogga, S., Weith, T., Aenis, T., Müller, K., Köhler, T., Härtel, L., \& Kaiser, D. B. (2014). WissenschaftPraxis-Transfer jenseits der ,Verladerampe“: Zum Verständnis von Implementation und Transfer im Nachhaltigen Landmanagement. Leibniz-Zentrum für Agrarlandschaftsforschung, Müncheberg.

Salet, W. (2014). The authenticity of spatial planning knowledge. European Planning Studies, 22(2), 293-305.

Stocker, A., \& Tochtermann, K. (2012). Wissenstransfer mit Wikis und Weblogs. Fallstudien zum erfolgreichen Einsatz von Web 2.0 in Unternehmen. Wiesbaden: Gabler.

Stringer, L. C., \& Dougill, A. J. (2013). Channelling science into policy: Enabling best practices from research on land degradation and sustainable land management in dryland Africa. Journal of Environmental Management, 114, 328-335.

Stützer, C. M., Köhler, T., \& Thiem, G. (2012). Formen der Kollaboration in Wissensnetzwerken. In T. Köhler \& N. Kahnwald (2012). Virtual Enterprises, Research Communities \& Social Media Networks. Proceedings of the GeNeMe 2012; Dresden, TUDPress.

UFZ Helmholtz-Zentrum für Umweltforschung (2009). Wissenschaftler im Dialog. Das Lernende Programm. Leipzig. Helmholtz-Zentrum für Umweltforschung.

UFZ Helmholtz-Zentrum für Umweltforschung (2011). Wissenschaftler im Dialog. Das Lernende Programm II. Leipzig. Helmholtz-Zentrum für Umweltforschung.

Wenger, E. (1998). Communities of Practice: Learning, Meaning, and Identity. Cambridge University Press.

Witzel, A. (1985). Problemzentriertes Interview. In G. Jüttemann (Hrsg.), Qualitative Forschung in der Psychologie: Grundlagen, Verfahrensweisen, Anwendungsfelder (S. 227-255). Weinheim: Beltz Verlag.

Zscheischler, J., Rogga, S. (2015). Transdisciplinarity in land use science - a review of concepts, empirical findings and current practices. Futures, 65, 28-44.

Zscheischler, J., Weith, T., Gaasch, N., Strauß, C., \& Steinmar, R. (2012). Nachhaltiges Landmanagement - eine kommunikative Herausforderung Jana; In: fub, Heft 5.

Zscheischler, J., Rogga, S., \& Weith, T. (2014). Experiences with Transdisciplinary Research. In: Systems research and behavioral Science. Wileyonlinelibrary. doi:101002/sres.2274. 